\title{
Fibrolamellar Carcinoma: A Distinct Variant of Hepatocellular Carcinoma That Is Still Surrounded by Unveils Mysteries
}

\author{
Consolato Sergi ${ }^{1,2^{*}}$ \\ ${ }^{1}$ Department of Laboratory Medicine and Pathology, University of Alberta, Edmonton, Canada \\ ${ }^{2}$ Department of Pediatrics, Stollery Children's Hospital, Edmonton, Canada \\ Email: sergi@ualberta.ca
}

Received 23 September 2014; revised 20 October 2014; accepted 14 November 2014

Copyright (C) 2014 by author and Scientific Research Publishing Inc.

This work is licensed under the Creative Commons Attribution International License (CC BY). http://creativecommons.org/licenses/by/4.0/

(c) (i) Open Access

\begin{abstract}
Fibrolamellar carcinoma (FL-HCC) is overall a quite rare variant of hepatocellular carcinoma (HCC) that is intriguing and poorly understood at same time. In humans, it affects essentially the young population (children and young adults) in the peculiar absence of underlying liver diseases. FLHCC has distinctive clinico-pathologic features with metastases frequently found in the regional lymph nodes, but also peritoneum and lungs. Although initially considered harboring an indolent course, survival outcomes have been recently updated pointing to a neoplasm concealing many aspects of biogenesis. In this review, morphology, molecular pathways, and prognosis of this rare tumor are presented.
\end{abstract}

\section{Keywords}

Liver, Tumor, Carcinoma, Pathogenesis, Differential Diagnosis, Therapy, Prognosis

\section{Introduction}

Fibrolamellar carcinoma (FLC/FL-HCC) is a quite rare variant of hepatocellular carcinoma (HCC) and is intriguing and poorly understood. Children, adolescents and youth are essentially the population affected by this tumor. Both sexes are involved and Caucasians are often the ethnics of this population. There is no history of parenchymal liver disease. In new pediatric books and literature [1], FL-HCC is reported as a distinct clinical and histologic variant representing almost 1/3 of all HCCs, which are diagnosed at pediatric age. Genetic hemochromatosis, tyrosinemia, alpha-1-antitrypsin deficiency as well as progressive familial intrahepatic cholestasis

${ }^{*}$ Corresponding author. 
(PFIC) or Byler's disease are often the underlying disorders at pediatric age harboring the risk to develop HCC, although all these conditions may show a cirrhotic rearrangement of the liver architecture [2]. Conversely, FLCC has a peculiar lamellar fibrosis, i.e. a fibrosis having a thickness, which is considered singular for this tumor and occurs in patients without liver cirrhosis or underlying liver disease. Laboratory markers for FL-HCC include serum neurotensin, vitamin B12 binding capacity (transcobalamin), and plasma des-gamma-carboxyprothrombin, which have been associated with disease burden. Serum alpha fetoprotein is elevated in only $10 \%$ vs. $60 \%$ of classic HCC. Clinically, FL-HCC is less than one tenth of all HCCs and patients with FL-HCC have similar symptoms to classic HCC (abdominal pain, ascites, hepatomegaly, obstructive jaundice, and systemic manifestations) and FL-HCC is found to be rarely associated with gynecomastia and Budd-Chiari syndrome. Xray shows a central scar, which looks like similar to the scar seen in presence of focal nodular hyperplasia (FNH), although is often calcified, which is uncommon with FNH.

\section{Gross Anatomy and Microscopy}

Grossly, FL-HCC is larger than conventional HCC and has a peculiar tendency to metastasize to regional lymph nodes. FL-HCCs are usually single, hard, scirrhous, and well-circumscribed. They are bulging and white-brown tumor on cut surface and show fibrous bands throughout and central stellate scar resembling a focal nodular hyperplasia. FL-HCC is the only liver tumor that is more common in left lobe, but may obviously involve both lobes. There may be variable bile staining, hemorrhage, and necrosis.

Light microscopy is used to make the diagnosis of this intriguing tumor. FL-HCC shows polygonal cells with abundant eosinophilic cytoplasm and large nucleoli. Thick fibrous collagen bands that at places encircle or surround partly the neoplastic cells (Figures 1-4) separate these cells. Additional histologic features comprise cytoplasmic pale $\mathrm{n} /$ bodies and copper depositions. Its most frequent differential diagnosis is the sclerotic variant of hepatocellular carcinoma, which does not harbor the characteristic feature of the fibrous collagen bands of large thickness as seen in FL-HCC. In the sclerotic variant thin collagen fibers are seen that may surround single cells, but thick fibrous collagen bands are not usually seen.

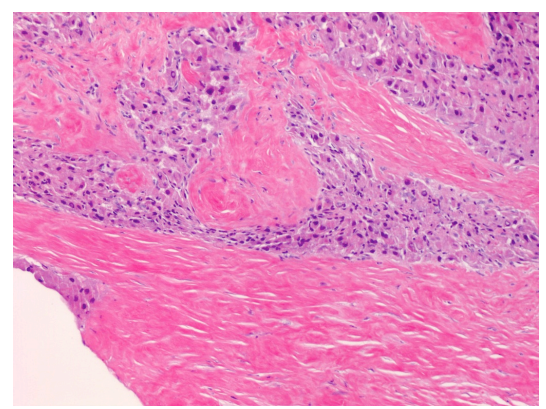

Figure 1. It shows thick fibrous collagen bands that at places encircle or surround partly the neoplastic cells (Hematoxylin \& eosin staining, $100 \times$ ).

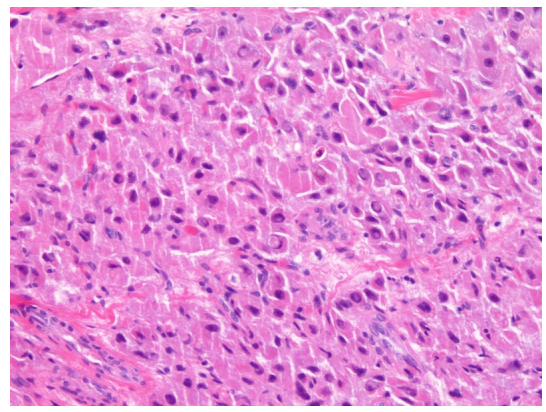

Figure 2. It shows polygonal cells with abundant eosinophilic cytoplasm and atypias (Hematoxylin \& eosin staining, 200×). 


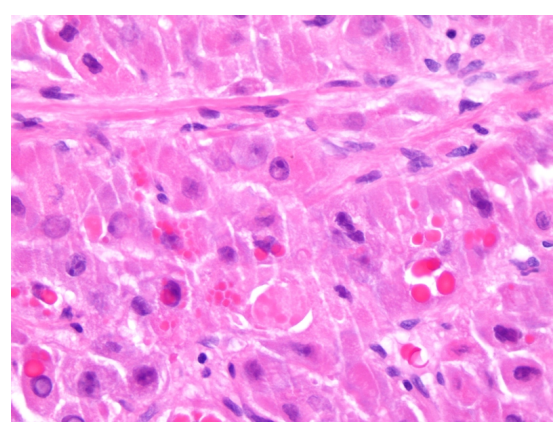

Figure 3. It shows polygonal cells with hyaline bodies (Hematoxylin \& eosin staining, 400×).

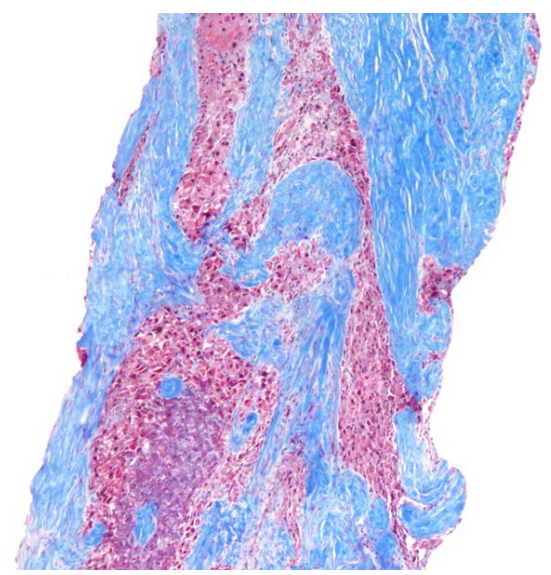

Figure 4. It shows how Masson's trichromic stain may highlight the thick fibrous collagen bands in FL-HCC (Masson's trichromic staining, $50 \times$ ).

Cells have abundant granular and intensely eosinophilic cytoplasm due to abundant mitochondria, which is a major characteristic of the electron microscopy investigation. Neoplastic cells have often pale bodies (ground glass cells) or periodic acid Schiff (PAS) positive hyaline globules [3].

It is important to remember that vascular invasion and necrosis are common and radiologic calcification corresponds exactly to necrosis with foreign body type reaction. Some variable features include focal nuclear pleomorphism, trabecular, adenoid or pelioid patterns and obviously conventional hepatocellular carcinoma may be found combined with FL-HCC. FL-HCCs show often metastases in the regional lymph nodes, but also peritoneum and lung.

Immunohistochemically, FL-HCC shows HepPar-1, cytokeratin 7 (Figure 5), epithelial membrane antigen (EMA), CD68, carcino-embryonic antigen (polyclonal antibody) or p-CEA, while there is usually no expression of alpha-fetoprotein (AFP), synaptophysin or chromogranin that are instead expressed in neuroendocrine markers. The presence of p-CEA in canalicular pattern is specific for HCC, probably due to cross reactivity to biliary glycoprotein I present in bile canaliculi of normal liver and hepatocellular neoplasms; monoclonal CEA (mCEA) is usually negative. The occurrence of such an immunophenotype has raised the hypothesis that FL-HCC may be a hepatobiliary hybrid neoplasia. Additional markers that are positive in FL-HCC include CAM5.2, AE1-3, and neurotensin, while inconstant expression is usually seen for alpha-1-antitrypsin (AAT). It seems that HepPar1, CK-7, EMA and CD68 are consistently expressed in the majority of FL-HCC. CK19 is usually negative or rarely positive (Figure 6) [4]. Other positive markers include fibrinogen (pale bodies), copper and copper-binding proteins, and bile that may be also easily observed using a negative iron stain (Prussian blue stain or Prussian Perl's stain).

Interestingly, Ang et al. pointed to the expression of epithelial growth factor receptor (EGFR), and Her-2. 
However, c-kit and estrogen and progesterone receptor were not demonstrated by these authors. Histology of the regional lymph nodes is mandatory and appropriate investigation will often show the same histologic pattern as observed in the liver (Figure 7). At ultrastructural level, FL-HCC shows cells with some resemblance to neuroendocrine tumors. Numerous mitochondria are, however, the best markers for FL-HCC. Pale bodies contain fibrinogen and are associated with intracytoplasmic luminal or bile canaliculi or, sometimes, accumulation of rough endoplasmic reticulum. Neoplastic cells may have dense core neuroendocrine-like granules, although neoplastic cells are not neuroendocrine markers positive.

Cytologically, FL-HCC is impressive showing very large, polygonal cells with abundant granular cytoplasm, prominent nucleoli in relatively large nuclei and some characteristic intracytoplasmic hyaline and pale inclusions as well as intranuclear inclusions (Figure 8). Thus, some pleural effusion cytology with these distinctive

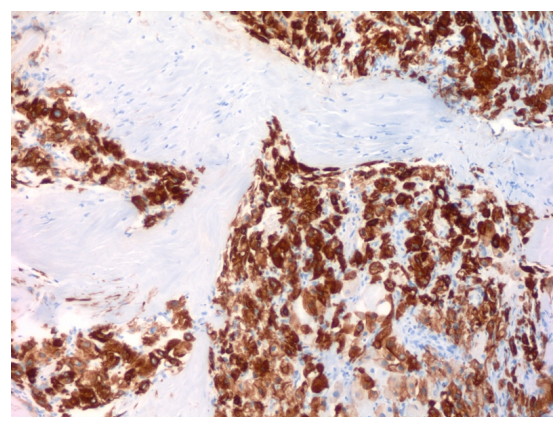

Figure 5. It shows how neoplastic cells are intensely stained with an antibody against cytokeratin 7 (Avidin-Biotin-Complex-ABC immunohistochemistry, 100×).

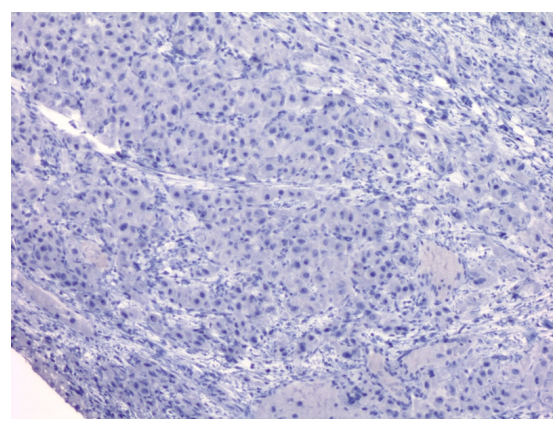

Figure 6. It shows how neoplastic cells are not stained with an antibody against cytokeratin 19 (Avidin-Biotin-Complex-ABC immunehistochemistry, 100×).

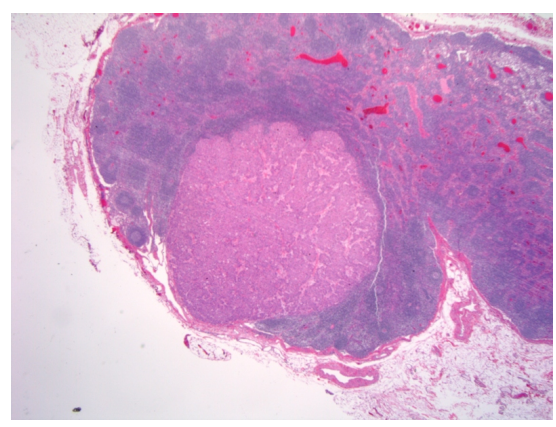

Figure 7. It shows a metastasis of FLHCC into a regional lymph node (Hematoxylin \& eosin staining, $12.5 \times$ ). 


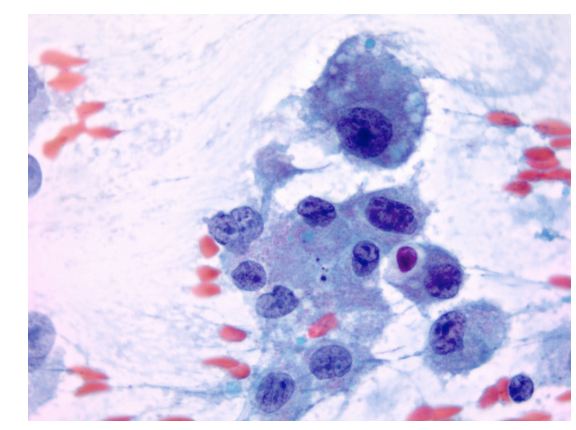

Figure 8. It shows the classic cytological pattern in a pleural effusion with very large, polygonal cells with abundant granular cytoplasm, prominent nucleoli in relatively large nuclei and some characteristic intracytoplasmic hyaline and pale inclusions as well as intranuclear inclusions (630×).

features in young adults presenting without cirrhosis, hepatitis B virus infection, and no history of alcohol abuse may suggest the right direction to make the appropriate diagnosis.

Differential diagnoses include adenosquamous carcinoma with sclerosis; cholangiocellular carcinoma, which is markedly glandular and mucin positive [5]; focal nodular hyperplasia, which has smaller size, contains bile ductular proliferating associated fibrous stroma and inflammatory cells, but no bile staining grossly and no hepatocyte atypia obviously; hepatocellular carcinoma, sclerosing variant, which shows no oncocytic change of the neoplastic cells, smaller size of tumor cells, and common pseudoglandular pattern; metastatic carcinoma with sclerotic stroma (history); other neuroendocrine tumors (neuroendocrine markers), and paraganglioma, which shows Zellballen growth pattern, round nuclei without atypia, vascular stroma but no dense fibrosis and, of course, positive for neuroendocrine markers [6]. Recently, a sub-variant of FL-HCC has been described, which is the clear cell variant of fibrolamellar carcinoma [7]. In addition to the classic FL-HCC, clear cells apparently due to ballooning and rarefactive changes of mitochondria have been described.

\section{Molecular Pathways}

The natural history of FL-HCC is poorly understood, but most recently specific molecular pathways seem to have identified some signatures that may peculiarly characterize this tumor. Interestingly, sometimes FL-HCCs may be initially classified as neuroendocrine tumors or conventional HCCs. However, typical pathways that are commonly mutated in conventional HCC, such as beta-catenin and p53, are not differentially regulated in FLHCC, but others including RAS, MAPK, EGFR, and PI3K have found to be upregulated in a small number of patients [8]. Riehle et al. have recently highlighted some pathways delineating that mechanistic target of rapamycin complex 1 (mTORC1) activation is found in FL-HCC and has been found associated with fibroblast growth factor receptor 1 (FGFR1) overexpression supporting the use of FGFR1 inhibitors and FGFR1 overexpression in patients with FL-HCC. Mitochondria are extremely represented in FL-HCC. Recently, we described the mitochondriome of cholangiocellular carcinoma [9]. Mitochondrial investigations on FL-HCCs should also be added to the research on FL-HCC. In fact, one aspect that is actually almost missing in research is the correlation of carcinogenesis with the mysterious role of mitochondrial genes.

\section{Prognosis and Management}

Recently, data from the "Fibrolamellar Carcinoma Consortium" have identified and updated overall survival of this rare tumor by using Kaplan-Meier methods. Cox regression models were used to assess the impact of different clinicopathologic variables on survival. Ang et al. reported that median age is 22 years, $86 \%$ being of Caucasian origin, and half of the patients presenting with stage IV disease. Females are slightly more often seen to be affected than males (58\% vs. $42 \%$ ). About $3 / 4$ of patients with FL-HCC undergo surgical resection and/or liver transplantation (LT). 31.5\% of these patients will receive perioperative therapy. Unresectable oncologic disease will drive patients to chemotherapy as well as patients who opt to be included in clinical trials. Recent 
therapy included sorafenib, which is a drug that has been co-developed and co-marketed by Bayer and Onyx Pharmaceuticals and is a drug approved for the treatment of advanced renal cell carcinoma, advanced hepatocellular carcinoma, and radioactive iodine resistant advanced thyroid carcinoma.

Median survival is 6.7 years. Ang et al. found that factors significantly associated with poor survival are female gender, advanced stage, lymph node metastases, macrovascular invasion, and disease, which is considered unresectable. Allan et al. queried the Surveillance, Epidemiology, and End Results (SEER) from 1973 to 2009 for all patients between age 0 and 19 with primary HCC. FL-HCC was seen exclusively in pediatric patients with age older than 5 years exhibiting greater survival compared to non-FL subtype (57\% vs. 28\%, respectively, $\mathrm{p}=0.002)$. SEER data indicate that tumor resection for children and adolescents with HCC significantly improved overall survival at 5 years compared to no surgery ( $60 \%$ vs. $0 \%$, respectively, $\mathrm{p}<0.0001$ ). Remarkably, and independently from the subtype, overall 5-, 10- and 20-year survival for the entire cohort was $24 \%$, $23 \%$, and 8\%, respectively (Allan B. J. et al., 2014). According to multivariate analysis, independent prognostic factors of lower mortality were surgical resection with a hazard ratio $(\mathrm{HR}=0.18)$, non-Hispanic ethnicity $(\mathrm{HR}=$ $0.52)$ and local disease at time of presentation $(\mathrm{HR}=0.46)$. Njei et al. (2014) reviewed recently prognosis of patients with FL-HCC vs. conventional HCC using both systematic review and meta-analysis. The authors included 17 studies involving 368 patients with FL-HCC and 9877 patients with conventional HCC. An overall statistically significant increase in the 5-year survival for the FL-HCC vs. the conventional HCC patients was found. Moreover, Njei et al. found also a significant increase in mean overall survival in patients with FL-HCC compared with the survival time of those with conventional HCC (84.9 \pm 15.8 vs. $42.9 \pm 6.5$ months) undergoing surgery (partial hepatectomy). However, there was no difference in patients undergoing liver transplantation.

The following information is aimed to delineate some management guidelines in more detail. In about three quarter of cases, patients undergo surgery and some LT too. Surgery is usually performed with curative intent, but palliative "debulking" resections are also carried out in some centers with advanced neoplastic disease and for determinate patients, who are not completely delineated in categories. Perioperative therapy includes preoperative chemotherapy, external beam radiation, and transarterial chemoembolization (TACE) with doxorubicin, cisplatin, and mitomycin-C. Postoperative adjuvant chemotherapy TACE, and/or radiation are also treatment choices. In single cases, transarterial radio-embolization with iodine-131-labeled lipiodol may be proposed. The choice of both pre- and postoperative chemotherapy is also sometimes carried out. In case of unresectable disease because of major blood vessel involvement, lymph node metastasis, and/or widespread M1 clinical staging, some choices may include various combinations of systemic therapy, with or without locoregional therapies including TACE, radiofrequency ablation, external beam radiation, percutaneous ethanol injection, and hepatic arterial infusion of cisplatin. Chemotherapy agents used in case of FL-HCC include fluoropyrimidines, doxorubicin, cisplatin, oxaliplatin, gemcitabine, and irinotecan. Interestingly, children with FL-HCC may undergo hepatoblastoma chemtherapy scheme. This scheme may include different combinations of cisplatin, doxorubicin, ifosfamide, vincristine, cyclophosphamide, etoposide, and fluoro-pyrimidines. In some occasions, thalidomide has been used. In the literature, it is also mentioned the use of the PIAF (cisplatin, interferom, doxorubicin, and 5-fluorouracil) regimen. Clinical trials aimed the evaluation of flavopiridol with cisplatin/irinotecan, the Chk inhibitor XL 844 plus gemcitabine, rapamycin, huang lian, the anti-endosialin antibody MORAb 004, and bland vs. doxorubicin-eluting bead chemoembolization. In other single cases, megestrol acetate, octreotide bevacizumab, EGFR tyrosin kinasse inhibitors, imatinib, and sorafenib have been used.

\section{Conclusion}

The liver is the third most common site for intra-abdominal malignancy in children after adrenal or paraspinal neuroblastoma and Wilms' tumor [10]. Of the malignant liver tumors, hepatoblastoma and hepatocellular carcinoma are most common and account for 2/3 of all liver neoplastic diseases. It seems that children of Hispanic ethnicity have higher rates of mortality, but HCC resection for curative intent significantly improves outcomes. Since 1956, from the first description of this tumor by Edmondson, there have been few progresses in research dealing with FL-HCC. In particular, very few advances have been done with respect to the molecular and genetic mechanisms that underlie its pathogenesis and behavior. FL-HCC expresses biliary, hepatocytic and hepatic-progenitor cell markers, and fewer genomic and epigenetic abnormalities than conventional HCC. The perception that FL-HCC is an indolent neoplastic disease that should be disgarded, because recent data point to high recurrence rates after surgery and considerably higher rates of morbidity, mortality and poor prognosis than 
thought. However, patients with FL-HCC treated with hepatic resection have significantly higher rates of 5-year survival than those with non-fibrolamellar carcinoma subtype of HCC. It is imperative to compare, in future studies, the efficacy of hepatic resection vs. LT in patients with FL-HCC and multi-institutional registries are important in compiling robust databases for efficient and efficacious clinical and translational research.

\section{References}

[1] Tanaka, Y., Inoue, T. and Horie, H. (2013) International Pediatric Liver Cancer Pathological Classification: Current Trend. International Journal of Clinical Oncology, 18, 946-954. http://dx.doi.org/10.1007/s10147-013-0624-8

[2] Fabbretti, G., Sergi, C., Consalez, G., Faa, G., Brisigotti, M., Romeo, G. and Callea, F. (1992) Genetic Variants of Alpha-1-Antitrypsin (AAT). Liver, 12, 296-301. Review.

[3] Torbenson, M. (2007) Review of the Clinicopathologic Features of Fibrolamellar Carcinoma. Advances in Anatomic Pathology, 14, 217-223. Review.

[4] Klein, W.M., Molmenti, E.P., Colombani, P.M., Grover, D.S., Schwarz, K.B., Boitnott, J. and Torbenson, M.S. (2005) Primary Liver Carcinoma Arising in People Younger than 30 Years. American Journal of Clinical Pathology, 124, 512-518. http://dx.doi.org/10.1309/TT0R7KAL32228E99

[5] Al-Bahrani, R., Abuetabh, Y., Zeitouni, N. and Sergi, C. (2013) Cholangiocarcinoma: Risk Factors, Environmental Influences and Oncogenesis. Annals of Clinical \& Laboratory Science, 43, 195-210.

[6] Corti, B., D’Errico, A., Pierangeli, F., Fiorentino, M., Altimari, A. and Grigioni, W.F. (2002) Primary Paraganglioma Strictly Confined to the Liver and Mimicking Hepatocellular Carcinoma: An Immunohistochemical and in Situ Hybridization Study. American Journal of Surgical Pathology, 26, 945-949. http://dx.doi.org/10.1097/00000478-200207000-00015

[7] Cheuk, W. and Chan, J.K. (2001) Clear Cell Variant of Fibrolamellar Carcinoma of the Liver. Archives of Pathology \& Laboratory Medicine, 125, 1235-1238.

[8] Kannangai, R., Vivekanandan, P., Martinez-Murillo, F., Choti, M. and Torbenson, M. (2007) Fibrolamellar Carcinomas Show Over-Expression of Genes in the RAS, MAPK, PIK3, and Xenobiotic Degradation Pathways. Human Pathology, 38, 639-644. http://dx.doi.org/10.1016/j.humpath.2006.07.019

[9] Bahitham, W., Liao, X., Peng, F., Bamforth, F., Chan, A., Mason, A., Stone, B., Stothard, P. and Sergi, C. (2014) Mitochondriome and Cholangiocellular Carcinoma. PLoS One, 9, e104694. http://dx.doi.org/10.1371/journal.pone.0104694

[10] Bulterys, M., Goodman, M.T., Smith, M.A., et al. (1999) Hepatic Tumors. In: Ries, L.A.G., Smith, M.A., Gurney, J.G., et al., Eds., Cancer Incidence and Survival among Children and Adolescents: United States SEER Program 1975-1995, SEER Program, NIH Pub. No. 99-4649, National Cancer Institute, Bethesda, MD, 91-98. 
Scientific Research Publishing (SCIRP) is one of the largest Open Access journal publishers. It is currently publishing more than 200 open access, online, peer-reviewed journals covering a wide range of academic disciplines. SCIRP serves the worldwide academic communities and contributes to the progress and application of science with its publication.

Other selected journals from SCIRP are listed as below. Submit your manuscript to us via either submit@scirp.org or Online Submission Portal.
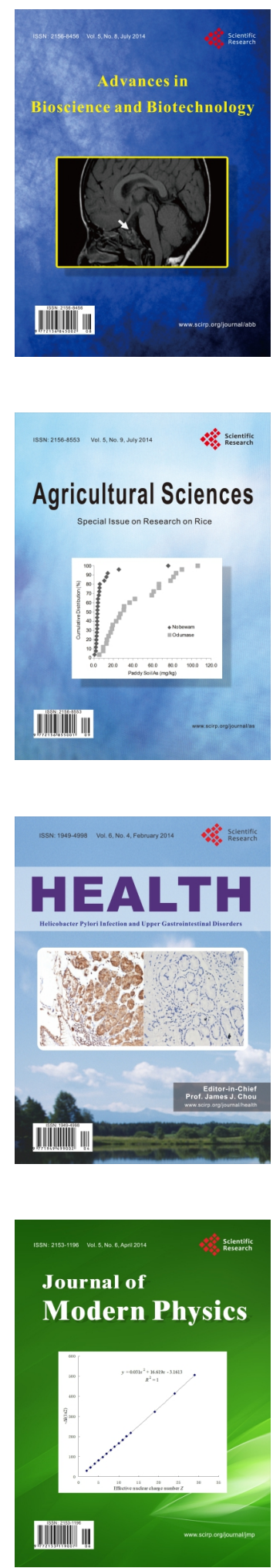
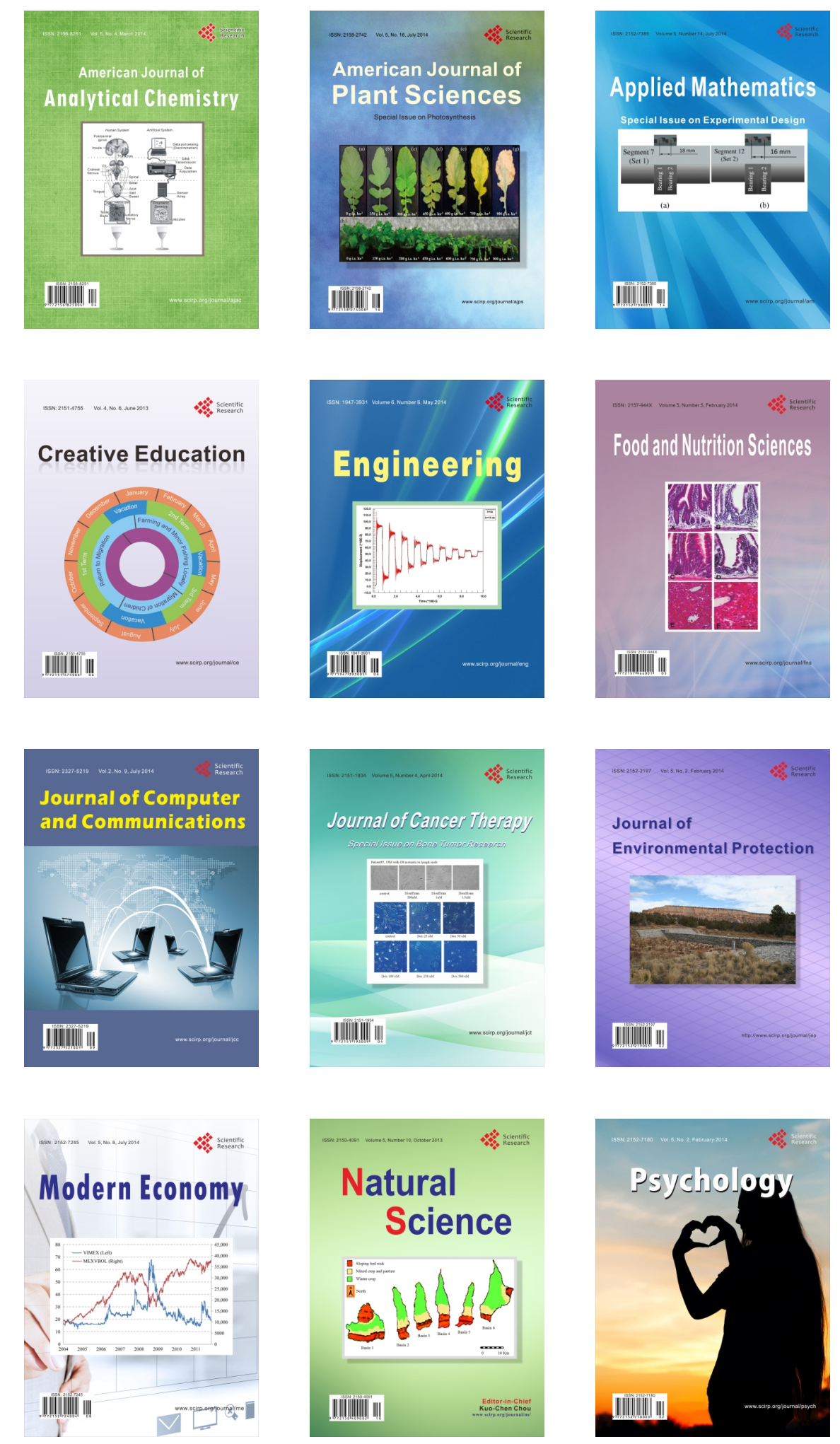